Images du travail, travail des images

1 | 2016

Quand les groupes professionnels se mettent en images

\title{
Baron Évelyne, Hersant Guy, Le Tirant Dominique, 2013, Pose Travail, Genk, Snoeck éditions, 280 p.
}

Jean-Paul Géhin

\section{OpenEdition}

\section{Journals}

Édition électronique

URL : http://journals.openedition.org/itti/1402

DOI : 10.4000/itti. 1402

\section{Éditeur}

Université de Poitiers

Référence électronique

Jean-Paul Géhin, «Baron Évelyne, Hersant Guy, Le Tirant Dominique, 2013, Pose Travail, Genk, Snoeck éditions, 280 p. », Images du travail, travail des images [En ligne], 1 | 2016, mis en ligne le 01 février 2016 consulté le 14 avril 2021. URL : http://journals.openedition.org/itti/1402 ; DOI : https://doi.org/ 10.4000/itti.1402

Ce document a été généré automatiquement le 14 avril 2021

Images du travail, travail des images 


\title{
Baron Évelyne, Hersant Guy, Le Tirant Dominique, 2013, Pose Travail, Genk, Snoeck éditions, 280 p.
}

\author{
Jean-Paul Géhin
}

\section{RÉFÉRENCE}

Baron Évelyne, Hersant Guy, Le Tirant Dominique, 2013, Pose Travail, Genk, Snoeck éditions, $280 \mathrm{p}$.

1 Voici un ouvrage original et élégant mariant livre d'art et essai en sciences sociales, et s'appuyant sur le croisement de trois points de vue :

2 Muséologique. À l'origine du projet, une commande du musée départemental de Seine et Marne, qui souhaite accorder une place importante au travail dans ce musée de société mais aussi accompagner le développement rapide du département en lien avec les cinq chantiers économiques et sociaux mis en œuvre par le Conseil général.

3 Photographique. Pour ce faire, le musée propose une résidence à Guy Hersant, photographe, spécialiste des portraits de groupe en Afrique puis sur le travail en France, notamment d'apprentis marins pour le musée port de Douarnenez ou d'habitants de logements collectifs à Parthenay. En 2011 et en 2012, il a réalisé 100 photographies de groupe dans 100 entreprises du département choisies en fonction de leur diversité et de leur représentativité de l'économie locale. Comme le note Évelyne Baron dans son introduction de l'ouvrage, «Il émane de ces photographies, à la fois une force et un respect du sujet, elles disent beaucoup sans être bavardes » (p. 10).

Ethnologique. Dans chacune de ces entreprises, Dominique Le Tirant, ethnologue et psychologue, spécialiste de la représentation des femmes au travail, a réalisé un entretien auprès d'un membre de l'entreprise, souvent un dirigeant, pour brosser son activité et son histoire, et dégager quelques caractéristiques du travail dans cette entreprise. Les entretiens ont fait l'objet d'un texte court témoignant en priorité de 
l'engagement de chacun au travail : «aspects sensibles relatifs à l'identité, à la transmission, à l'éthique du métier, à la relation au collectif de travail » (p.18).

Image 1.

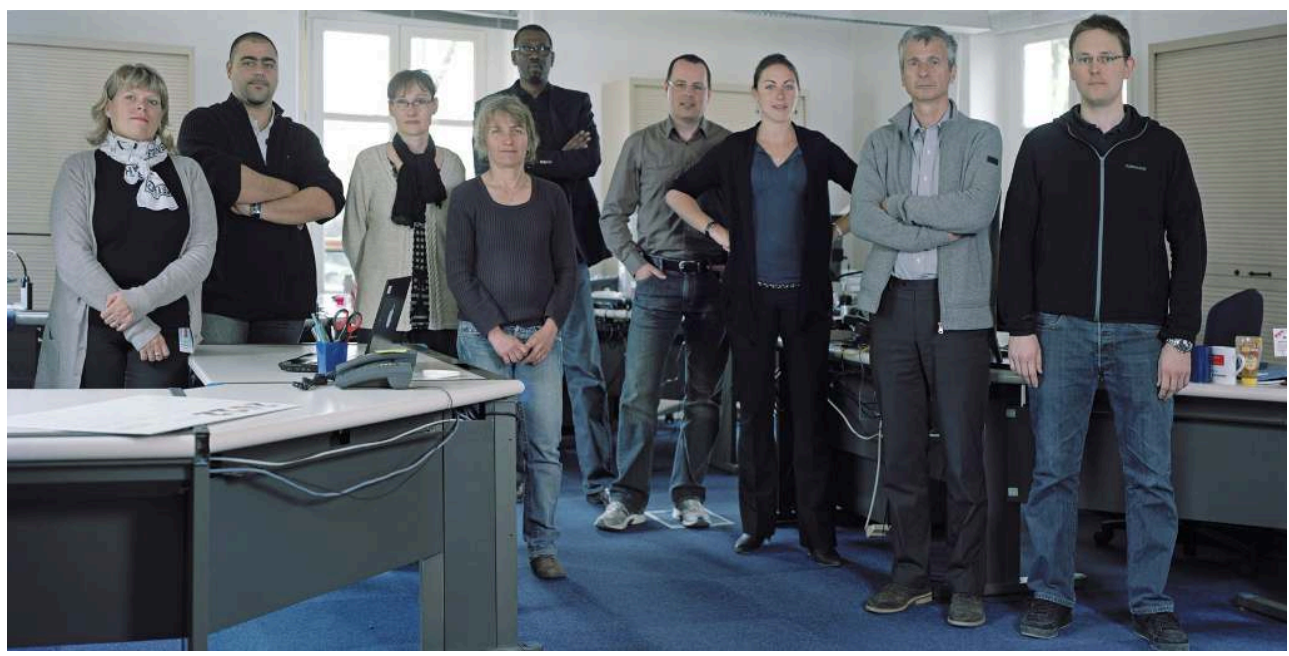

5 Entretien et photographie sont placés face à face. Pour chaque entretien, sont précisés le lieu, la date de réalisation ainsi que le nom et les fonctions de la (ou des) personne(s) interviewée(s). Chaque photographie est légendée présentant les individus ${ }^{1}$ par leur métier, leur fonction, quelquefois leur statut : contractuel, intérimaire, propriétaire, stagiaire, apprenti... Le couple photographie-résumé de l'entretien forme le cœur de l'ouvrage et lui donne un caractère systématique. On se retrouve face à un répertoire des métiers et des entreprises en Seine et Marne, une sorte d'atlas local du travail. En tous cas, pour reprendre l'expression de Howard Becker, une forme originale de construction de représentations du monde social ${ }^{2}$.

6 Les 100 séquences textes images sont organisées, selon les auteurs, en fonction des aspects sensibles de l'activité, soit 6 grandes parties (les gestes de métier, la parole et l'écoute, le travail au corps, le collectif de travail, les cartographies mentales, les identités de métier), elles même souvent subdivisées en sous parties introduites par un verbe d'action (toucher-manipuler, voir, écouter, parler, éprouver, s'émouvoir, faire corps, se représenter, hériter-transmettre, s'engager). Le lecteur reste bien souvent dubitatif par rapport à cette organisation thématique. Pourquoi tel couple texte image dans telle catégorie? Qu'est ce qui justifie par exemple que les métallurgistes, les fromagers, l'ingénieur énergie, les salariés d'une agence immobilière, les employés d'une grande surface de bricolage, les éditeurs de logiciel, l'équipe d'animation d'un château médiéval, un médecin et des infirmières se retrouvent ensemble sous la thématique écouter?

7 L'ouvrage fait l'objet d'une introduction à trois voix (conservatrice, photographe, ethnologue) et est rythmé par des analyses proposées par des spécialistes de sciences sociales du travail (sociologue, historien, philosophe, psychologue) et deux écrivaines. On a ainsi dans les mains un bel ouvrage articulant textes et images, points de vue scientifiques et approches artistiques. 


\section{Une subtile mise en scène}

8 En esquissant une rapide histoire de la photographie de travail, Pierre Gaudin nous rappelle que le travail de Guy Hersant s'inscrit dans une double tradition :

9 Celle de la photographie de travail qui apparait dès la mise en place du procédé durant le XIX ${ }^{e}$ siècle permettant tant de saisir les activités industrielles naissantes que de conserver les gestes, les pratiques, les contextes et les métiers traditionnels de la civilisation rurale en train de disparaître. Au tournant du siècle avec Eugène Atget en France, Jacob Riis ou Lewis Hines aux États-Unis, la photographie va en priorité montrer la misère sociale, les conditions de vie et de travail des ouvriers dans un monde en train de s'urbaniser. Considérée alors comme un témoignage "objectif », un " élément de preuve ", elle aura un impact social considérable et contribuera à modifier les représentations et la législation. Il faudra attendre les années 1920, avec l'allégement du matériel et l'émergence de nouvelle conception de la réalité et de l'objectivité, pour que s'affirme ce que Pierre Gaudin appelle une véritable photographie industrielle au double sens du terme «de sujet industriel en photographie (hommes, machines, lieux, produits, mouvements) et d'art industriel, désormais reproductible à grande échelle, léger, transportable et hyperactif» (p.158). Nait ainsi la photographie documentaire, parallèlement au documentaire de création au cinéma, affirmant un regard personnel engagé et assumant une certaine subjectivité.

10 Celle du portrait de groupe qui est un genre très courant et codifié dont chacun a une expérience intime à travers la photographie de classe, celle de mariage ou encore de régiment. Le portrait de groupe offre un éclairage sociologique mis en lumière par les analyses d'Yvette Delsault ${ }^{3}$ et, dans le champ du travail, par la démarche du sociologue et photographe Lewis W. Hines documentant le travail des enfants aux États Unis au début du XXe Siècle. Très récemment, le travail d'Arnaud Théval, photographiant des classes de lycéens professionnels, accompagné par des commentaires de Sylvain Maresca, montre l'intérêt sociologique d'une telle approche $e^{4}$.

11 L'ouvrage Pose travail nous informe sur le travail contemporain en opérant un déplacement par rapport aux approches traditionnelles des activités économiques et sociales : "Créer autour du monde du travail, «travailler le travail» du point de vue reculé et décalé de l'artiste, de l'écrivain, du cinéaste est essentiel pour aider à mieux comprendre ce que travailler veut dire " (Pierre Gaudin, p. 156). Il évite un point de vue trop unilatéral soit critique, soit hagiographique ou publicitaire. Le choix de la photographie de groupe implique une mise en scène réalisée par le photographe (composition, éclairage, conduite d'acteurs ...) en lien avec les photographiés ${ }^{5}$ qui ne peuvent pas être considérés comme passifs. Ils interviennent en nous informant par maints détails comme les regards, la façon de positionner les mains, la tenue des corps, les vêtements qu'ils portent, les objets qu'ils mettent en valeur...

12 Cette (plutôt ces) mise(s) en scène ne signifie(nt) pas production factice ou artificielle. $\mathrm{Au}$ contraire, elle(s) permet(tent) d'appréhender le travail dans son quotidien, dans ce qu'il contient à la fois de plus banal ${ }^{6}$ et de plus fondamental. C'est saisir l'image que les acteurs veulent donner de leur travail. C'est "mesurer dans le regard de ceux qui nous regardent leur implication propre: consentement, soumission, résistance, usure, colère, révolte, organisation, acquisition, transmission" (Pierre Gaudin, p. 160). C'est donc une mise en scène négociée entre photographe et photographiés que nous donne à voir Pose travail, d'autant plus que les textes qui accompagnent les photographies ont aussi fait l'objet 
d'une validation par l'entreprise, entrainnant quelquefois des demandes de corrections significatives.

Au-delà des régularités imposées par le photographe et le genre portrait de groupe, l'analyse attentive de ces cent photographies et des textes associés permet de souligner quelques points saillants. Notons d'abord trois fausses photographies de groupe, constituées d'une collection de photographies individuelles, à deux ou à trois, regroupées sur la même page: cela semble logique pour les sœurs bénédictines fabricant des santons en terre cuite puisque leurs vœux de silence les incitent à des activités solitaires; cela apparaît plus énigmatique pour les travailleurs sociaux de la maison départementale des solidarités de Coulommiers ou les professionnels de la justice du tribunal de grande instance de Meaux. Faut-il y lire une étonnante absence de collectif dans ces professions? Soulignons ensuite que dix portraits ne mettent en scène qu'un professionnel: ce qui s'explique lorsqu'il s'agit d'artisan ou de salarié travaillant seul: "faiseur de livres ", ingénieur énergie, entraîneuse de natation, traductrice, professeure d'équitation, constructeur de maison en bois; ce qui reste inexpliqué pour l'enseignant en collège ou les trois salariés de la SNCF présentés isolément.

Image 2.

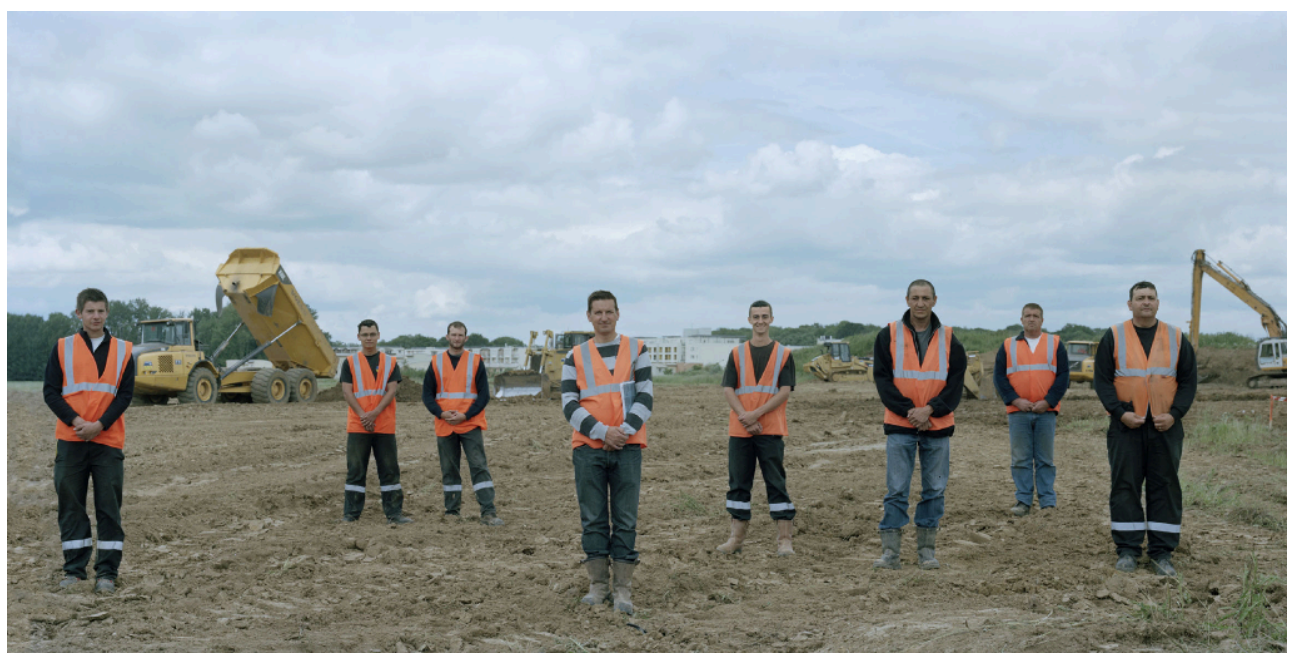

Concernant les photos de groupe, la mise en scène renvoie d'abord au travail du photographe qui a l'initiative et est engagé dans une approche systémique. Il a négocié le lieu et le moment avec la direction de l'entreprise, installé le matériel de prise de vue et réalisé un véritable travail de direction d'acteurs, qui est précisé en introduction: "Commence alors un travail de positionnement des individus dans l'espace du cadre (...) Le photographe s'intéresse ensuite aux postures, incitant les personnes à se tenir toujours droit et à se redresser. Il porte une grande attention à la disposition des mains (...) En dernier lieu le photographe s'intéresse à l'expression des visages. C'est là le plus difficile: le photographe recherche la neutralité des expressions et prescrit l'effacement des sourires trop épanouis » (p. 21).

D'autres éléments de la mise en scène échappent peu ou prou au photographe :

- Le lieu de la photographie qui est rarement le lieu de travail lui-même (atelier, chaîne, chantier) mais se situe en périphérie de l'espace de production : entrée des locaux, extérieur 
de l'entreprise, magasin, décor considéré comme typique et photogénique par la direction de l'entreprise et/ou les photographiés.

- Les photographiés sont exceptionnellement assis (3 cas), ils se touchent que très rarement (4 cas).

- Assez fréquemment, ils tiennent fièrement un outil, plus souvent symbolique de la profession ou du statut qu'usuel : outils traditionnels pour les ouvriers; plans, feuilles, cahiers pour les responsables; pelle et pioche pour les archéologues, pèse bébé pour la puéricultrice, violon pour les musiciens, oiseau de proie pour les fauconniers, sac de caisse pour les caissières de supermarché...

- Quelquefois des éléments de protection (casque, masque, lunettes, gilet...)

- Plus rarement des signes distinctifs de l'entreprise (badge, tee-shirt, parka...)

\section{Le groupe et les individus}

Cet ouvrage documente non seulement le travail contemporain mais aussi nous renseigne sur le travail photographique dans la mesure où photographies comme textes proposent une approche réflexive sur la production d'images du travail. Il organise une rupture avec les images traditionnelles, en sortant de l'espace et du temps du travail concret, en instituant une pose, qui malgré le jeu de mots tentant n'est pas vraiment une pause : pas de cigarettes ici, pas de machines à café, pas de détente, pas de corps relâchés, pas de travailleurs assis (ou si rarement). Si on ne travaille pas, on simule le travail, on ne se repose pas non plus, on joue au sens théâtral du terme en réalisant souvent de savantes mises en scène.

17 En opérant un déplacement d'un enregistrement a priori immédiat du travail en train de se faire vers une photographie de groupe fortement et clairement mise en scène par l'ensemble des acteurs, Pose travail interroge la possibilité de filmer, de photographier le travail. Est-il représentable? Directement? Ou faut-il opérer un détour? Et si oui lequel? En plagiant une analyse de François Niney $^{7}$ sur le rapport au réel des documentaires de création, on peut affirmer qu'il y a du réel dans ces photographies de Pose travail au moins deux éléments : ce que le photographe et/ou les photographiés ont voulu y mettre ; ce qui leur échappe, ce qu'ils ne contrôlent mais qui se retrouve dans la photo comme par inadvertance, une sorte de réel en contrebande. 
Image 3 .

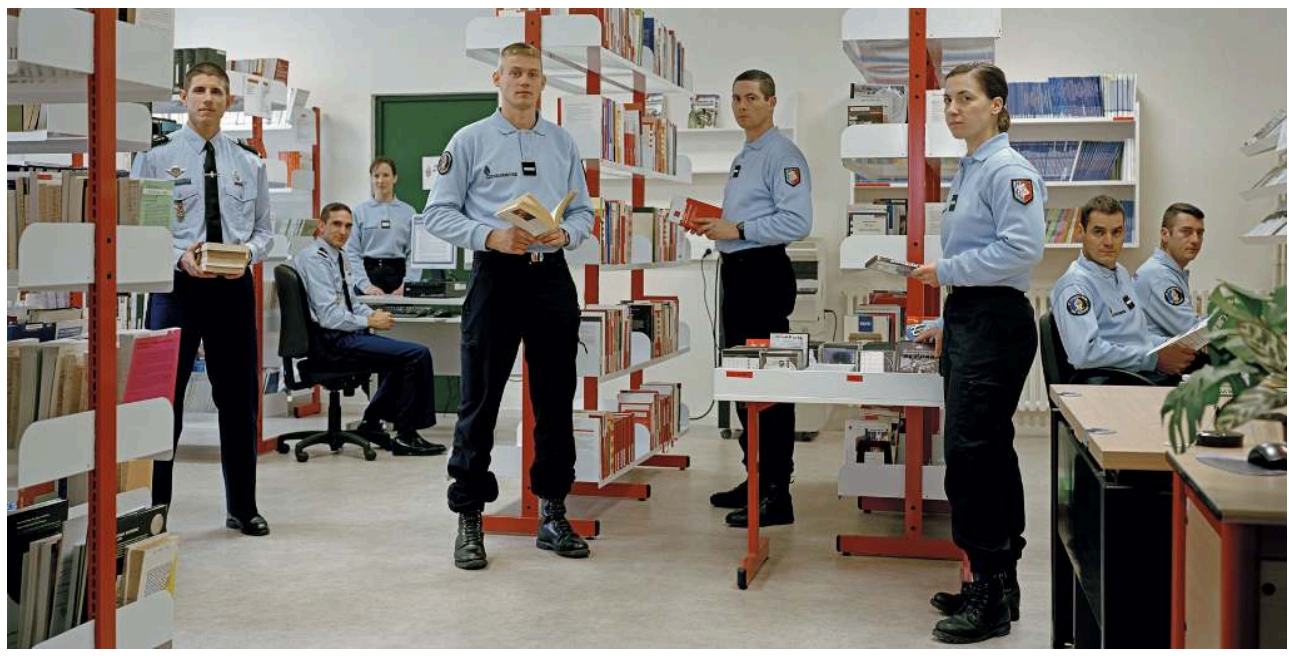

Bien que Pose travail mette en scène des collectifs organisés de manière systématique par le photographe et sans doute orchestrés en sous-main, dans bien des cas, par les directions ou les services communication des entreprises, il nous montre d'abord des individus. Des individus, certes contraints par des organisations mais disposant d'une marge de manœuvre et d'autonomie, d'une façon personnelle de se tenir, de manier des outils, de revêtir les habits de travail et les éléments de protection, de s'approprier l'espace de la photo, et surtout de regarder droit dans les yeux le spectateur...

19 À sa manière Guy Hersant rend compte de la montée des individualités mais aussi des formes d'articulations étroites individus/collectifs qui se tissent au quotidien dans l'exercice du travail contemporain. Et se faisant, il nous donne à voir ce que Morgan Jouvenet nomme les formes nouvelles de l'engagement dans le travail: "Contre l'uniforme, les arrangements avec la tenue écrivent une partition mêlant enthousiasme, usure et défiance. Ainsi les collectifs à l'image (et les images des collectifs) sont enrichis des individualités - qui restituent ici l'humaine contingence de leur composition - et laissent imaginer au spectateur le jeu qu'elle introduit dans le fonctionnement quotidien » (p.72).

La photographie est devenue une pratique sociale banale et les individus en maitrisent, peu ou prou, quelques codes, même si c'est de manière peu consciente. Ils se plient à l'exercice imposé de pose mais ne se fondent pas dans le collectif. Ils donnent à voir des éléments de leur personnalité. Des individus certes engagés dans leur travail mais gardant des distances. Ceci est d'autant plus net que les groupes sont plus petits. Les cadres serrés rapprochent les corps, réduisent la profondeur de champ et mettent l'accent sur des individualités singulières : regards, postures, mimiques, manières de porter un vêtement de travail ou un élément de protection, tenues du corps... Autant d'indices, maigres mais récurrents, de la place de l'individu dans le groupe, de sa capacité à négocier sa participation.

\section{Les rapports sociaux du travail}

Les photographies de groupe permettent de déplacer le regard, en évitant l'impossible et vaine saisie directe du travail concret dans l'espace de production sous forme de gestes, de techniques, de savoir-faire... qui ne sont souvent qu'apparence et qui cachent l'essentiel : les relations interindividuelles, les hiérarchies formelles ou informelles, les 
rapports de domination, bref, l'ensemble des rapports sociaux de production, qui constitue le cœur du travail industriel dans le système capitaliste libéral. Constat déjà réalisé dans les années 1930 par Bertolt Brecht ${ }^{8}$ :

"Ce qui complique encore la situation, c'est que moins que jamais la seule reproduction de la réalité ne dit quoique ce soit sur cette réalité. Une photographie des usines Krupp ou de AEG ne nous apprend pratiquement rien sur ces institutions. La réalité proprement dite a glissé dans son contenu fonctionnel. La réification des relations humaines, par exemple à l'usine, ne permet plus de les restituer. Il faut donc effectivement construire quelque chose d'artificiel, de posé »

La modernité de cette pensée et son application à Pose travail sont tout à fait étonnantes. Les portraits de groupe montrent, par petites touches et comme par inadvertance, ce qui est généralement invisible, voire caché par les et aux acteurs du travail : la séparation nette entre travail et hors travail, le poids et la prégnance de la hiérarchie malgré la mise en scène de collectifs unis et homogènes, la permanente préoccupation qu'ont les institutions, professions ou entreprises de leur image.

Image 4. Famille Bompart, viticulteurs. Saisonniers et viticulteurs propriétaires exploitants. Dernier jour de la vendange. Vendanges 2011. Saâcy-sur-Marne.

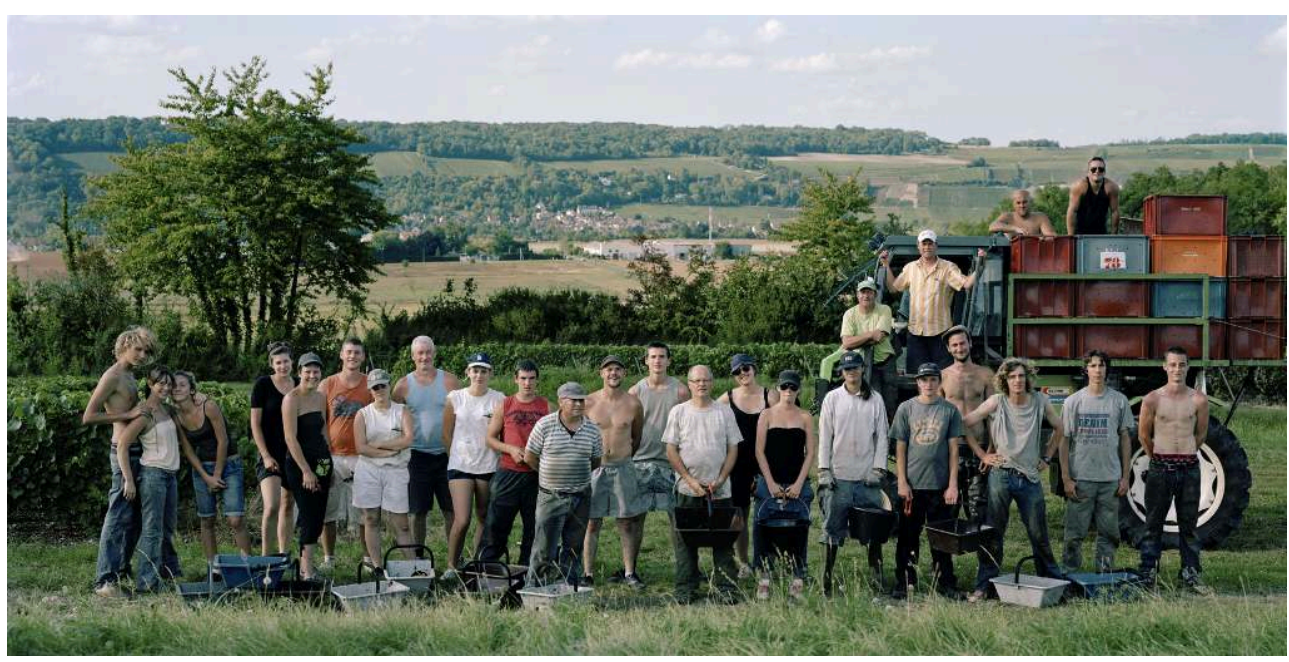

Photo : Guy Hersant.

Même si ces photographies ne nous montrent pas le travail en train de se faire, elles se situent bien dans le temps et l'espace de l'entreprise et de la production, et nous disent peu sur la vie hors travail des individus photographiés. Seuls les non-salariés mettent en scène des éléments de leur vie domestique en faisant poser leurs enfants : c'est le cas d'Amandine, gérante d'une SARL qu'elle a créée et qui pose aux milieux de ses créations (rouleaux de tissu) en portant fièrement dans son dos sa fille Alice qui a environ un an (p.255) ; c'est aussi celui d'un viticulteur (p.181) ou d'un maraîcher (p.269) qui posent avec familles, salariés et enfants ; ou encore, de ce couple d'agriculteurs qui mettent en scène leurs salariés et leurs enfants : au centre le mari, à sa droite sa famille, à sa gauche «ses » salariés (p.233). On notera ici que se combinent, et c'est souvent le cas, hiérarchie sociale et hiérarchie de genre, sa femme et ses filles étant reléguées à la périphérie de la photo alors que le jeune fils, ostensiblement protégé par le bras de son père, se retrouve au centre. Plus courant encore, des non-salariés pose avec un animal domestique, généralement un chien (pp. 123, 185, 205, 237). 
Image 5. Planète Chanvre. Usine de transformation. Mécanicien, chef de ligne et chef de site, manutentionnaire, agricultrice et associée. Jouarre. Printemps 2012.

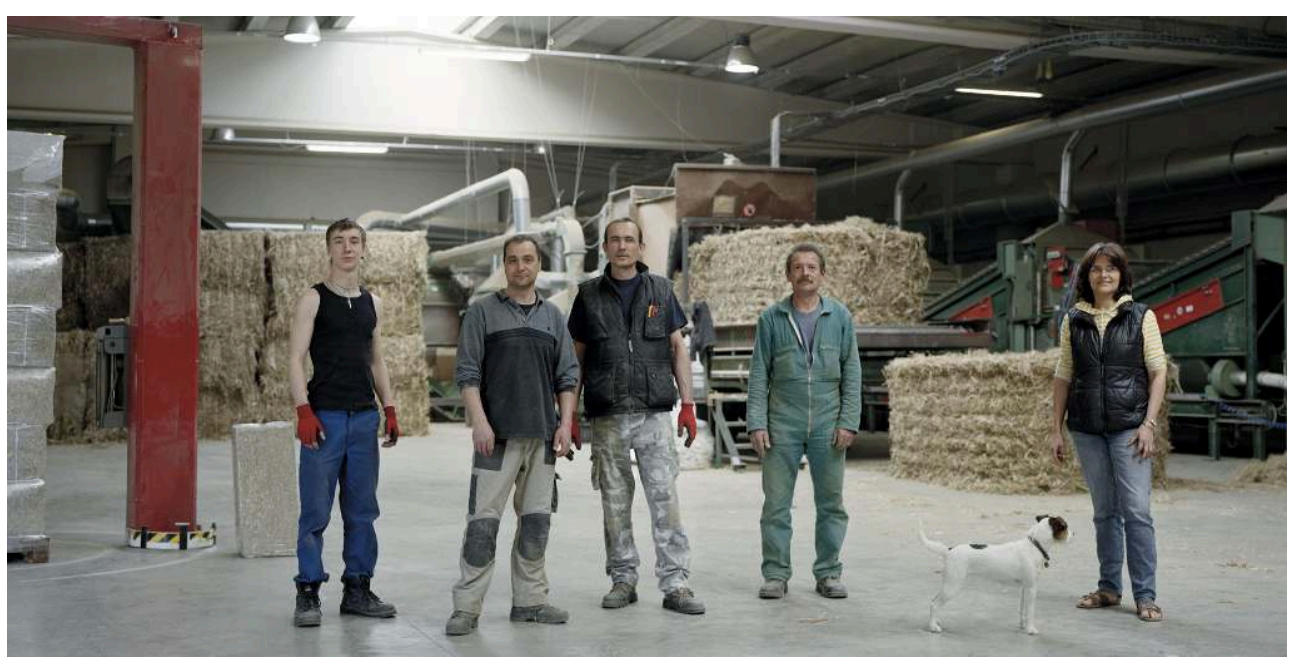

Photo : Guy Hersant. sociales entre les différents membres de l'entreprise : ainsi cette entreprise privée de traitement de déchet (p.67) qui fait figurer ses neuf salariés en affichant clairement leur métiers, leurs fonctions et leur place dans l'organisation. En extérieur, sur fond de l'entreprise et de tas de matériaux à recycler, se distinguent quatre groupes : au centre et au premier plan, le responsable du site et le président, en habits de ville mais sans cravate, sont séparés des autres salariés par deux éléments verticaux forts en arrièreplan (château d'eau et grande borne) ; à leur droite, tout à fait à gauche de l'image et au deuxième plan, la secrétaire et encore plus en retrait le chauffeur ; à leur gauche, dans le même plan que le chauffeur, deux groupes de deux salariés d'exécution (grutier, manutentionnaire, plus loin grutier, acheteur) séparés entre eux par une pelleteuse, et facilement identifiables par leurs bottes et leurs vêtements de travail ; entre les deux, approximativement sur le même plan que la secrétaire, l'agent commercial, portant comme lui impose sa fonction costumes cravates.

Cet affichage limpide constitue une forme d'exception, la plupart des portraits de groupe mettent en scène une sorte de proximité sociale autour de l'idée de collectif unis. La liste des photographiés donne rarement leur position sur la photo. Les indices de hiérarchies sociales sont plus subtils et ténus mais ils n'en restent pas moins présents à ceux qui veulent bien les déchiffrer. 
Image 6. Château de Vaux-le-Vicomte.

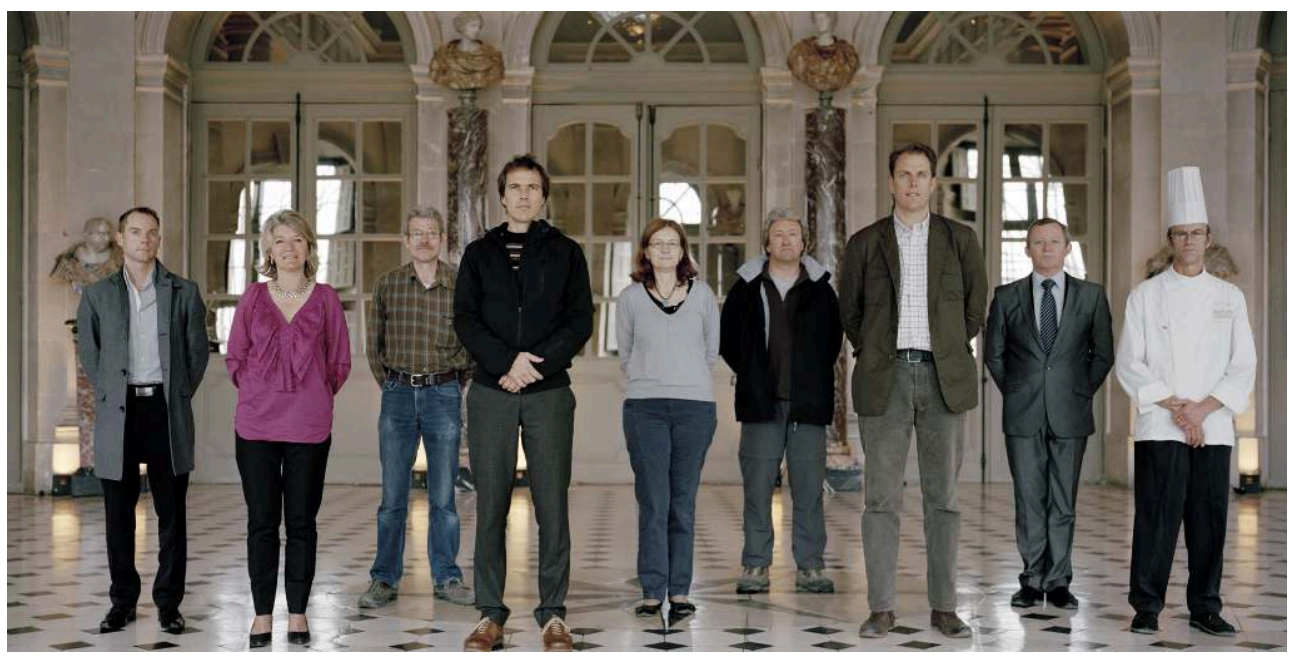

Équipe de responsables : responsables de la boutique, responsable d'entretien, responsable de la comptabilité, chef jardinier, Jean-Charles de Vogüé et Alexandre de Vogüé, propriétaires, chef cuisinier, chef maître d'hôtel, responsable événementiel. Grand salon, printemps 2012.

La photo de l'équipe des responsables du château de Vaux le Vicomte (p.203) est de ce point de vue exemplaire et rappelle les jeux de devinettes que proposaient aux enfants les images d'Épinal : où se trouve le jardinier? Où est caché le cuisinier? Le cadre est serré, rapprochant les corps des 9 photographiés, réduisant la profondeur de champ et ne laissant voir du décor que trois monumentales portes vitrées. Les fonctions des photographiées ne sont pas données par ordre de figuration dans la photo. Malgré cette mise en scène, les hiérarchies sociales éclatent: notons d'abord, le hors champ composé des sans doute très nombreux salariés d'exécution; remarquons ensuite que l'on reconnaît sans difficulté les deux propriétaires, les seuls dont la légende donne le nom, les sortant de l'anonymat des autres figurants : ils sont habillés décontractés et sans cravate, vêtus différemment, séparés et pas tout à fait au centre; pourtant leur position au premier plan, leur taille et leur prestance ne laissent aucun doute. Concernant les salariés, ils adoptent tous une position de recul et de soumission; on reconnaît facilement le chef cuisinier et le chef maître d'hôtel, on pense identifier la responsable événementiel et on hésite pour les quatre derniers responsables, d'ailleurs un peu plus en retrait : le jean et la chemise à carreau ne désignent-ils pas les probables origines ouvrières du responsable d'entretien? L'anorak et les chaussures de marche, le chef jardinier?

Dans nombre de photographies, quelques indices permettent de dépasser les affichages et les non-dits, de lire les hiérarchies et les distances sociales : net contraste dans ce chantier naval entre ouvriers et employés (p.235); chefs très souvent au centre et au premier plan; impression de vacances des propriétaires, qui sont les seuls à ne pas tenir des objets ou des outils de travail (p.65); tenue du corps particulière (p.237); absence de blouse (p.111) (p.49); absence d'outil (p.41); mise en avant de symboles d'une activité intellectuelle ou de conception comme le stylo (p.45; p. 173), la photo, le carnet (p.49; p. 109 ; p. 241). Ce sont souvent de subtiles distinctions qui font le cadre, de responsable, le propriétaire ou le dirigeant. Ainsi le professeur est le seul à ne pas porter de cravates rouges dans le groupe des secondes bac pro restauration (p.133); de même, c'est le chapeau qui fait le directeur (p.89); ou encore, l'absence d'outils et de casques de protection qui fait le responsable (p.103) 

d'entreprise impose une tenue décontractée pour tous (filiale française d'un grand éditeur américain de logiciels p. 87) (fournisseur d'accès à Internet, p. 221), soit que les circonstances (inventaire) font que chacun est habillé en détendu lors de la photo et effacent les signes distinctifs des différents métiers (p.85), soit au contraire que les uniformes ou les vêtements professionnels (p.35; p. 51 ; p. 135 ; p. 141 ; p. 149)) rendent plus difficile la lecture des différences sociales : ainsi, dans cette agence immobilière (p. 83) sont tenu à un code couleur strict, homme comme femme, haut jaune, bas noir, estce la directrice qui déroge à la règle en portant une robe jaune ? Certaines mises en scène avec casques, gilets et divers éléments de protection peuvent également jouer ce rôle (p.167; p. 169 ; p. 207). veulent donner d'eux-mêmes les professions ou les entreprises. C'est le cas de la photographie de cette fromagerie familiale (p.79) mettant en avant ses fromages et faisant de ses salariés, masqués dans des blouses et coiffes blanches, de simples garants, anonymes et interchangeables, de l'hygiène et de la qualité des produits. La photo en une de l'ouvrage est un autre exemple de stratégie de communication d'entreprise : les salariés de cet établissement d'embouteillage d'eau de source sont présentés comme stables et connaissant des promotions. L'équipe photographiée est "composée de compagnons fidèles et motivés par cet esprit familial de compagnonnage» (p.270). La photo véhicule mais aussi nuance ce message. L'alignement des 6 salariés en habits de travail sur fond de bouteilles empaquetées suggère esprit d'entreprise, hygiène et soin apporté au traitement des eaux. Cependant, on distingue facilement le chef d'équipe et l'air intimidé des six photographiés vêtus de coiffes et de blouses blanches laisse comme une impression de malaise, sans doute en partie liée au fait que ces manuels ne savent comment occupés leurs mains inoccupées le temps de la photo et que les blouses de protection ne sont pas adaptées aux corps des salariés.

Dans d'autres cas, c'est l'image de la profession qui est en jeu: ainsi les élèves de sixième année de l'école supérieur d'ostéopathie donne à la fois une image de sérieux et d'application, notamment à travers les regards et les classeurs, et d'individualité et d'esprit d'initiative grâce à la mise en scène d'un savant désordre (p.125). De même la photo en habit de cérémonie des élèves officiers de gendarmerie (p.151) met délibérément l'accent sur l'ordre, la tradition, le prestige tout en suggérant quelques éléments de modernité avec les filles au premier rang. Reste que la présence de l'officier de la guardia civil, en introduisant une touche supplémentaire d'exotisme, rend moins crédible l'ensemble.

31 Les groupes professionnels peuvent chercher à modifier leur image comme c'est le cas des officiers de gendarmerie (p.2) «surpris» dans le centre de documentation à "effectuer des travaux de recherche universitaire dans le cadre de leur master " nous dit la légende mais la mise en scène trop soignée, les positions trop figées laissent le spectateur dubitatif. De même, ces quatre dirigeants d'une entreprise de roulements à bille sont des salariés d'un établissement d'un grand groupe qui a fait l'objet d'un plan de reprise d'entreprise par ses salariés: alignés devant leur bureau, affichant clairement titres et fonctions, très droits, vêtements ajustés, presque rigides, ils mettent l'accent sur leur rigueur et leurs compétences de gestion dans leur nouveau statut. 

montre l'exemple de ce lycée agricole (p.145) qui propose deux photographies juxtaposées, représentant deux groupes d'élèves, les uns en formation professionnelle scolaire, les autres en apprentissage. Tout présente une grande proximité entre ces deux filières : les vêtements des élèves, presque tous revêtus de grandes combinaisons de travail; le décor, identique, suggérant l'alternance école entreprise puisque un grand tableau d'école recouvre la grande moitié gauche de l'image, laissant la place à droite à ce qu'on peut assimiler à une porte de hangar. Un détail cependant retient l'attention et remet en cause l'idée d'équivalence entre les filières, le visage des élèves, quelquefois bronzé et majoritairement féminin pour la filière scolaire, entièrement blanc et masculin pour l'apprentissage, suggérant alors origines et destinées sociales différenciées.

$\mathrm{Au}$ total, Pose travail explore des chemins peu pratiqués et propose des formes renouvelées de représentations sociales du travail contemporain. Certes, l'ouvrage n'est pas sans limite, le caractère institutionnel de certains textes et images pose problèmes et implique un travail de décryptage. Mais une voie est ouverte. Le détour par la pose et la mise en scène permet de déplacer le point de vue... Et de contourner l'obstacle maintes fois souligné du travail difficilement saisissable, filmable, photographiable. Il y a en tout cas ici une méthode: "LA CARTE EST PLUS INTÉRESSANTE QUE LE TERRITOIRE » pour reprendre le titre d'une exposition du personnage principal du roman de Houellebecq ${ }^{9}$, qui d'ailleurs est un photographe et peintre qui réalise l'essentiel de son œuvre en représentant le travail contemporain.

\section{NOTES}

1. Dans bien des cas interviewé(s) et photographié(s) ne sont pas les mêmes, l'entretien étant bien souvent réalisé auprès d'un dirigeant alors que les photographies mettent en scène couramment les salariés d'exécution.

2. Becker Howard, 2008

3. Yvette Delsaut, La Photo de classe, in Carnet de sociologie, 1988, pp 83-96

4. Voir « Moi, le groupe »

5. A noter qu'il n'est pas toujours simple de faire le départ dans la mise en scène entre ce qui est le fait du photographe et celui des photographiés.

6. L'infra-ordinaire, si cher à Georges Pérec.

7. Niney François, 2002, L'épreuve du réel à l'écran; Essai sur le principe de la réalité documentaire. $2 \mathrm{e}$ édition, Bruxelles, de Boeck

8. Bertold Brecht, 1931, Le procès de l'opéra de quat'Sous, cité par Pierre Gaudin, p.155

9. Houellebecq Michel, 2010, La carte et le territoire, Paris, Flammarion 